\title{
Mutation-in-Brief
}

\section{A Novel Mutation of the Steroidogenic Acute Regulatory Protein (StAR) Gene in a Japanese Patient with Congenital Lipoid Adrenal Hyperplasia}

\author{
Katsura Ishizu ${ }^{1}$, Junko Tsubaki ${ }^{1}$, Wakako Jyo ${ }^{1}$, Toshihiro Tajima ${ }^{1}$ \\ ${ }^{I}$ Department of Pediatrics, Hokkaido University School of Medicine, Sapporo, Japan
}

\section{Introduction}

Congenial lipoid adrenal hyperplasia (CLAH) is the most severe form of congenital adrenal hyperplasia. It is characterized by impaired synthesis of all the adrenal steroids including mineralocorticoids, glucocorticoids, and sex steroids (1). Affected individuals are phenotypically female and have severe salt wasting. This disease is especially frequent in the Japanese population $(1,2)$.

The cause of this disease is the genetic defects of the gene for steroidogenic acute regulatory protein (StAR). So far, more than 20 different mutations in the StAR gene have been found in patients with CLAH from various ethnic groups (1-5). In the present study we analyzed the StAR gene in a $46, \mathrm{XX}$ female patient with CLAH and found one novel mutation.

\section{Methods and Patient}

\section{Genetic analyses}

Informed consent for DNA analysis was obtained from the patient's parents. The ethical committee of our university permitted this study. To analyze the StAR gene, genomic DNA was

Received: August 15, 2007

Accepted: October 4, 2007

Correspondence: Dr. Toshihiro Tajima, Department of Pediatrics, Hokkaido University School of Medicine, N15, W7, Kita-Ku, Sapporo 060-8638, Japan

E-mail: tajeari@med.hokudai.ac.jp obtained from white blood cells by standard procedures. The exons and exon-intron boundaries of the StAR gene were amplified by polymerase chain reaction (PCR) using oligonucleotide primers as described in a previous report (3). These PCR products were purified and directly sequenced using an automated DNA sequencer (Applied Biosystems, Inc., Foster City CA).

\section{Patient's report}

The girl patient was the first child of unrelated healthy Japanese parents. She was delivered at term after an unremarkable gestation through cesarean section because of breech presentation. Her birth weight was 3,184 g. She was noticed to have hyperpigmentation and failure to thrive at $14 \mathrm{~d}$, and was referred to our hospital at $27 \mathrm{~d}$ of age. On physical examination she had remarkable pigmentation and normal female external genitalia with no ambiguity. Her body weight had decreased (3,045 g). The laboratory and endocrinological data are summarized in the Table 1 . The electrolytes were within normal ranges. Endocrinological examination showed a markedly high plasma adrenocorticotropin stimulating hormone (ACTH) level (3,341 pg/ml). The serum 17-hydroxyprogesterone level was normal. On ultrasonography, bilateral adrenal glands were slightly enlarged (right $1.4 \times 1.9 \mathrm{~cm}$, left $1.6 \times$ $1.2 \mathrm{~cm}$ ) (Fig. 1A). The patient's karyotype was $46, \mathrm{XX}$. She was diagnosed as having adrenal 
Table 1 Biochemical findings of the patient with CLAH

\begin{tabular}{lll}
\hline & Patient & Normal range \\
\hline $\mathrm{Na}(\mathrm{mEq} / \mathrm{L})$ & 134 & $135-145$ \\
$\mathrm{~K}(\mathrm{mEq} / \mathrm{L})$ & 5.5 & $3.5-4.8$ \\
$\mathrm{ACTH}(\mathrm{pg} / \mathrm{ml})$ & 3341.87 & $47.9 \pm 22.8$ \\
Cortisol $(\mu \mathrm{g} / \mathrm{dl})$ & 10.74 & $9.1 \pm 4.8$ \\
$17-\mathrm{OHP}(\mathrm{ng} / \mathrm{ml})$ & 0.3 & $0.9 \pm 1.01$ \\
Plasma aldosterone $(\mathrm{ng} / \mathrm{dl})$ & 232.4 & $38.12+20.95$ \\
Plasma renin activity $(\mathrm{ng} / \mathrm{ml} / \mathrm{h})$ & 44.91 & $8.58 \pm 6.61$ \\
\hline
\end{tabular}

A
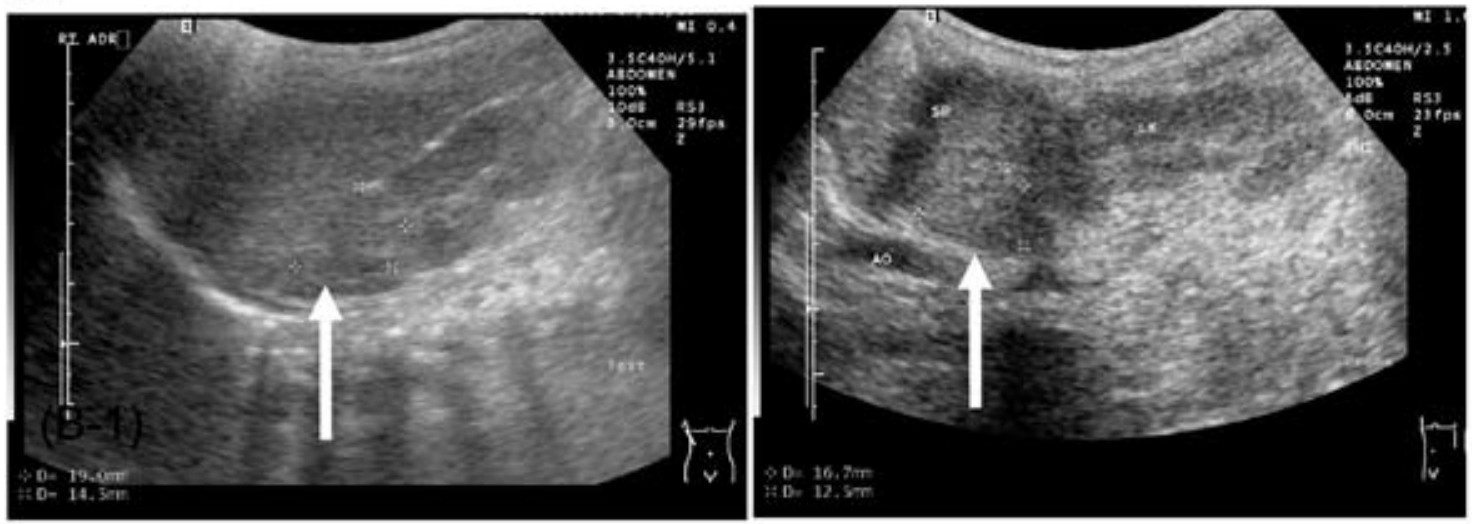

B

$(B-1)$

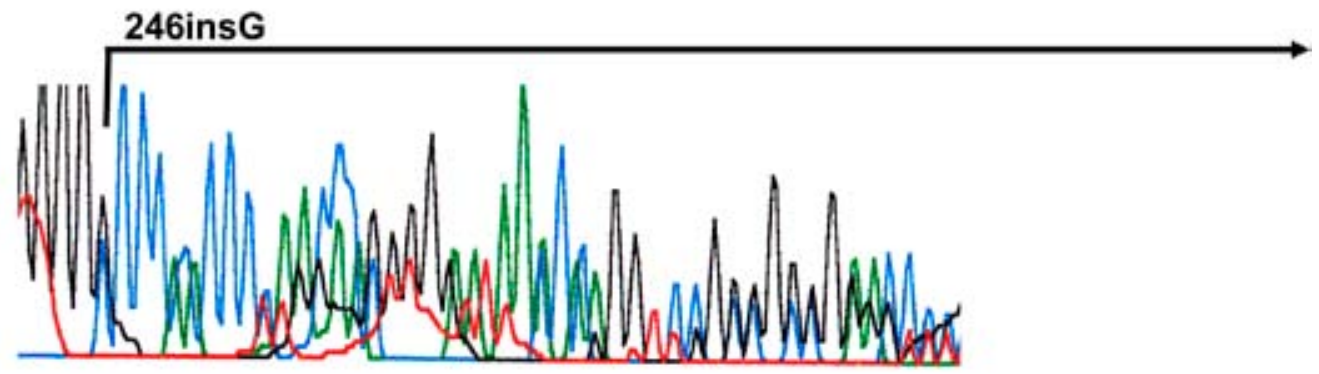

(B-2)

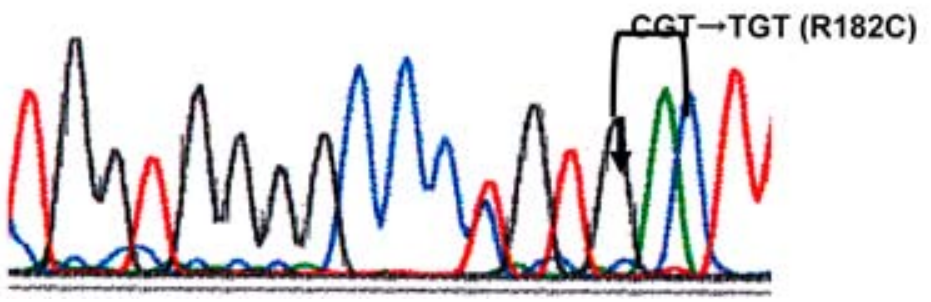

Fig. 1 A: Ultrasonography of adrenal glands. B: Sequence analysis of the patient 1. (B-1) The patient had a one base insertion (246insG) as a heterozygous state. Note overlapping signals after the insertion site. (B-2) An arrow indicates the $\mathrm{C}$ to $\mathrm{T}$ transition. This change substitutes cysteine for arginine at codon 182. 
insufficiency caused by CLAH. She was treated successfully with hydrocortisone and fludrocortisones and has grown well.

\section{Results}

The sequencing analysis of the patient revealed two heterozygous mutations (Fig. 1B). One mutation was 246 insG in exon 2 (Fig. 1B-1). The other, designated R182C, was caused by a $\mathrm{C}$ to $\mathrm{T}$ transversion at the first nucleotide of codon 182 in exon 5 (Fig. 1B-2). The insertion mutation was paternally transmitted and $\mathrm{R} 182 \mathrm{C}$ was maternally transmitted, indicating autosomal recessive inheritance.

\section{Discussion}

The patient has a novel R182C mutation in one allele and a previously reported 246insG mutation in the other. Nakae et al. (3) reported one Japanese CLAH patient who has a homozygous frameshift mutation of 246insG. This frame shift mutation caused a premature stop codon in exon 2 . We did not determine the functional consequence of the R182C mutation. As reviewed previously, the StAR missense mutations that cause CLAH are all clustered in exons 5-7. The missense mutation at codon 182 (R182L) has been frequently identified among Palestinian Arabs (2) and the R182L mutation has also been identified in one Japanese patient. In addition, arginine at 182 is present in the putative lipid transfer domain of StAR by structural modeling. Thus, R182C might be the cause of CLAH in our patient.

Differential diagnosis between CLAH and adrenal hypoplasia is sometimes difficult. In the presence of normal female genitalia, an XY karyotype suggests CLAH. In the case of 46, XX female, massive adrenal enlargement is a classical feature of CLAH $(1,2)$. Our patient showed moderately enlarged adrenals, suggesting CLAH. It is of note that a lack of radiologically demonstrable adrenal hyperplasia has been described in CLAH patients (5).Thus, genetic analysis of the StAR gene is useful for diagnozing CLAH.

Finally, 46, XX CLAH patients show spontaneous puberty, however their ovaries are at risk of the developing cysts and torsion $(4,5)$. Because of this, it is important to confirm the diagnosis of CLAH in 46, XX patients by gene analysis.

\section{References}

1. Fujieda K, Okuhara K, Abe S, Tajima T, Mukai T, Nakae J. Molecular pathogenesis of lipoid adrenal hyperplasia and adrenal hypoplasia congenita. J Steroid Biochem Mol Biol 2003;85:483-9.

2. Bose HS, Sugawara T, Strauss JF 3rd, Miller WL. The pathophysiology and genetics of congenital lipoid adrenal hyperplasia. International Congenital Lipoid Adrenal Hyperplasia Consortium. N Eng J Med 1996;335:1870-8.

3. Nakae J, Tajima T, Sugawara T, Arakane F, Hanaki K, Hotsubo T. Analysis of the steroidogenic acute regulatory protein (StAR) gene in Japanese patients with congenital lipoid adrenal hyperplasia. Hum Mol Genet 1997;6:571-6.

4. Fujieda K, Tajima T, Nakae J, Sageshima S, Tachibana K, Suwa S, et al. Spontaneous puberty in 46 , XX subjects with congenital lipoid adrenal hyperplasia. Ovarian steroidogenesis is spared to some extent despite inactivating mutations in the steroidogenic acute regulatory protein (StAR) gene. J Clin Invest 1997;99:1265-71.

5. Bose HS, Sato S, Aisenberg J, Shalev SA, Matsuo N, Miller WL. Mutations in the steroidogenic acute regulatory protein (StAR) in six patients with congenital lipoid adrenal hyperplasia. J Clin Endocrinol Metab 2000;85:3636-9. 